\title{
Le rapport à la littérature des enseignants de français : enjeux et pratiques
}

\section{Marie-Christine Beaudry et Sylvain Brehm}

\section{(2) OpenEdition}

\section{Journals}

Édition électronique

URL : http://journals.openedition.org/trema/4510

DOI : $10.4000 /$ trema.4510

ISSN : 2107-0997

Éditeur

Faculté d'Éducation de l'université de Montpellier

Édition imprimée

Date de publication : 1 octobre 2018

Pagination : 1 - 3

ISBN : 979-10-96627-05-9

ISSN : $1167-315 \mathrm{X}$

Référence électronique

Marie-Christine Beaudry et Sylvain Brehm, « Le rapport à la littérature des enseignants de français : enjeux et pratiques », Tréma [En ligne], 49 | 2018, mis en ligne le 01 novembre 2018, consulté le 24 septembre 2020. URL : http://journals.openedition.org/trema/4510 ; DOI : https://doi.org/10.4000/ trema.4510

Ce document a été généré automatiquement le 24 septembre 2020

Trema 


\title{
Le rapport à la littérature des enseignants de français : enjeux et pratiques
}

\author{
Marie-Christine Beaudry et Sylvain Brehm
}

Les différents travaux menés sur le rapport au savoir, notamment en sociologie (Bourdieu et Passeron, 1966), en recherche clinique (Beillerot, 1989), en microsociologie (Charlot, 1997) et en anthropologie (Chevallard, 1992) témoignent de la pertinence de son étude dans plusieurs champs de recherche et de la pluralité des perspectives à partir desquelles l'envisager. En didactique du français, différentes recherches sont menées depuis maintenant deux décennies sur le "rapport à », dans la foulée des travaux du groupe ESCOL (Bautier, 2001; Charlot, 1997). Barré-De Miniac (2002: 3) propose une définition éclairante du rapport à l'écriture, qu'elle décrit comme un «ensemble hétérogène, fait de conceptions, de représentations, d'attitudes et de valeurs». Des travaux comme ceux d'Émery-Bruneau (2010) ont porté plus spécifiquement sur le rapport à la lecture littéraire de futurs enseignants au secondaire québécois et ont mis en lumière les plans personnel et didactique que comporte ce rapport. Ainsi, nous connaissons mieux le rapport à l'écrit (Barré-De Miniac, 2000, 2002; Chartrand et Prince, 2009), le rapport à la culture (Simard et Falardeau, 2007) et le rapport à la lecture et à la lecture littéraire (Émery-Bruneau, 2010 ; Ulma et Winkler, 2010). Si le concept nous semble s'être relativement stabilisé en didactique du français, les principales recherches ont porté surtout sur les enseignants ou futurs enseignants du niveau secondaire. Des zones d'ombre existent, particulièrement dans le rapport à la littérature des (futurs) enseignants, par exemple sur un possible continuum ou sur des ruptures entre les différents ordres d'enseignement. L'impact de ce rapport sur les pratiques d'enseignement, notamment des textes poétiques (Brillant Rannou, 2010), dramatiques (Bernanoce, 2013) ou multimodaux (Lacelle, Lebrun et Boutin, 2012), demeure largement méconnu. Ce numéro thématique cherche à prolonger ces travaux en apportant un éclairage sur des aspects moins connus du rapport à la lecture et aux textes littéraires des enseignants et des futurs enseignants en France et au Québec. 
2 Ana Dias-Chiaruttini présente sa conception du « rapport à », qu'elle construit à la croisée des réflexions d'inspiration psychanalytique et sociologique. Elle examine, par la suite, les apports de cette notion, mais aussi les questions que son usage soulève en didactique du français. Elle conclut en montrant comment l'analyse du rapport à la littérature et de son enseignement permet de mieux connaître ce qui, justement, s'enseigne sous le nom de littérature.

3 Les contributions suivantes portent plus spécifiquement sur l'enseignement de la littérature au Québec. Julie Babin et Olivier Dezutter se sont intéressés au rapport à la lecture d'une œuvre, sur le plan didactique, de trois professeurs de français au niveau collégial. Ils ont filmé leurs pratiques en classe et ont réalisé un entretien avec chacun d'eux. Leur recherche fait apparaître l'importance des tensions ressenties par ces enseignants entre les exigences ministérielles et leur propre vision des finalités de la lecture scolaire d'une œuvre littéraire.

4 Judith Émery-Bruneau, quant à elle, étudie les conceptions de la poésie des enseignant-e-s de français du secondaire québécois afin de comprendre les objectifs qu'ils assignent à l'enseignement des textes poétiques et de connaître les objets, les corpus et les tâches privilégiés dans les séquences didactiques ou les séances consacrées à la poésie. Sa recherche révèle que la plupart des enseignants entretiennent une conception traditionnelle de la poésie et de son enseignement qui ne tient pas vraiment compte des évolutions de ces formes poétiques, notamment depuis la fin du XIX ${ }^{e}$ siècle.

5 Marion Sauvaire et Alexandra Gagné, pour leur part, présentent les résultats d'une étude exploratoire fondée sur la mise en œuvre d'un dispositif de formation destiné à susciter chez de futurs enseignants de littérature du collégial l'appropriation de modèles d'enseignement de la lecture littéraire. Pour ce faire, elles ont demandé à ces futurs praticiens de tenir un journal de lecture afin de les amener à adopter une posture réflexive sur leurs pratiques de sujets lecteurs. Les résultats obtenus suggèrent que l'écriture réflexive constitue un moyen efficace pour favoriser l'appropriation de modèles didactiques de la lecture littéraire.

6 La contribution de Sylvain Brehm apporte également un éclairage sur la formation d'étudiants qui souhaitent enseigner le français au collégial, ainsi qu'au secondaire. Cependant, elle porte plus particulièrement sur la conception de la littérature québécoise contemporaine de ces futurs enseignants. L'article révèle ce que représente pour eux "la littérature québécoise contemporaine ", quels critères de sélection des œuvres ils entendent choisir et quelles finalités ils assignent à l'enseignement de ces textes.

7 L'article de Marie-Christine Beaudry, Jill Vandermeerschen, Alexie Miquelon, Sylvie Marcotte et Joannie Dubois présente une partie des résultats d'une recherche visant à décrire le rapport à la lecture littéraire des futurs enseignants québécois du primaire, du secondaire et du collégial. Les résultats font ressortir deux types de rapport chez les répondants. Ils font également ressortir quelques variables qui semblent déterminantes pour structurer le rapport à la lecture littéraire des sujets de leur enquête. 


\section{BIBLIOGRAPHIE}

Barré-De Miniac, C. (2000). Le rapport à l'écriture. Aspects théoriques et didactiques. Villeneuve d'Ascq : Presses Universitaires du Septentrion.

Barré-De Miniac, C. (2002). « Du rapport à l'écriture de l'élève à celui de l'enseignant ». Recherches et éducations, $\mathrm{n}^{\circ} 2$, 3e trimestre 2002, http://rechercheseducations.revues.org/index283.html.

Bautier, É. (2001). Pratiques langagières et scolarisation. Revue Française de Pédagogie, 137, 117-161.

Beillerot, J. et al. (1989). Savoir et rapport au savoir. Élaborations théoriques et cliniques. Paris : Éditions Universitaires.

Bernanoce, M. (2013). Le répertoire théâtral dans son contexte scolaire, à l'épreuve des genres et des esthétiques, Le Français aujourd'hui, n 180, 2013, p. 27-38.

Brillant Rannou, N. (2010). Le poème et son lecteur. Lire en poésie : expérience littéraire et enjeux pour l'enseignement du français en lycée. Thèse inédite. Université Rennes 2.

Bourdieu, P. et Passeron, J-C. (1966). Les héritiers. Paris : Minuit.

Charlot. B. (1997). Du rapport au savoir. Paris : Anthropos.

Chartrand, S-G. et Prince, M. (2009). La dimension affective du rapport à l'écrit d'élèves québécois. Canadian journal of Education, 32/2, pp. 317-343.

Chevallard, Y. (1992). Concepts fondamentaux de la didactique : perspectives apportées par une approche anthropologique. Recherches en didactique des mathématiques.12/1, pp. 73-112.

Dezutter, O. et Morissette, C. (2010). Que pensent des élèves québécois des œuvres à lire en contexte scolaire et des activités imposées autour de ces lectures? In Du corpus scolaire à la biblioth èque intérieure (B. Louichon et A. Rouxel, dir.). Rennes : Presses universitaires de Rennes, pp. 201-209.

Émery-Bruneau, J. (2010). Le rapport à la lecture littéraire. Des pratiques et des conceptions de sujets-lecteurs en formation à l'enseignement du français à des intentions didactiques. Thèse de doctorat. Université Laval.

Lebrun, M, Lacelle, N. et Boutin, J.-F. (2012). La littératie médiatique multimodale : de nouvelles approches en lecture-écriture à l'école et hors de l'école. Montréal : Presses de l'Université du Québec.

Simard, C. et Falardeau, É. (2007). Le rapport à la culture des enseignants de français et son rôle dans l'articulation de la culture avec les contenus disciplinaires. In E. Falardeau, C. Fisher, C. Simard et N. Sorin (dir.). La didactique du français. Les voies actuelles de la recherche (p. 147-163). Sainte-Foy : Pul.

Ulma, D. et Winkler, I. (2010). « L'enquête IMEN-Littérature : vers une comparaison francoallemande du rapport des futurs enseignants au canon littéraire », Nouveaux Cahiers d'AllemandRevue de linguistique et de didactique, 28/1, pp. 19-46. 
AUTEURS

MARIE-CHRISTINE BEAUDRY

Université du Québec à Montréal

SYLVAIN BREHM

Université du Québec à Montréal 